\title{
Caracterización química de biocarbón de ápices de caña de azúcar elaborado mediante carbonización hidrotérmica y adición de catalizadores orgánicos Chemical characterization of biochar from sugarcane tips produced by hydrothermal carbonization and addition of organic catalysts
}

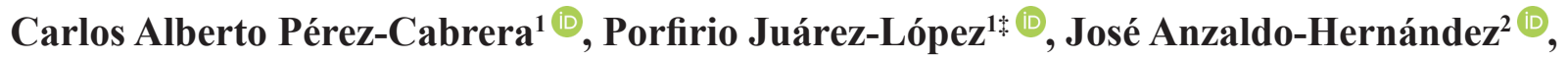 \\ Irán Alia-Tejacal ${ }^{1}{ }^{(D)}$, Eduardo Salcedo-Pérez ${ }^{3}{ }^{\mathbb{D}}$, Dagoberto Guillén-Sánchez $^{1}{ }^{(\mathbb{D})}$, \\ Rosendo Balois-Morales ${ }^{4}{ }^{\circledR}$, Víctor López-Martínez ${ }^{1}{ }^{\mathbb{D}}$ y Rogelio Castro-Brindis $^{5}$ (D)
}

\footnotetext{
${ }^{1}$ Posgrado en Ciencias Agropecuarias y Desarrollo Rural. Universidad Autónoma del Estado de Morelos. Avenida Universidad 1001.62210 Cuernavaca, Morelos, México.

*Autor para correspondencia (porfirio.juarez@uaem.mx)

${ }^{2}$ Universidad de Guadalajara, Centro Universitario de Ciencias Exactas e Ingenierías. Blvd. Marcelino García Barragán 1421, Olímpica. 44430 Guadalajara, Jalisco, México.

${ }^{3}$ Universidad de Guadalajara, Centro Universitario de Ciencias Biológicas y Agropecuarias. Ramón Padilla Sánchez 2100, Nextipac. 45200 Zapopan, Jalisco, México.

${ }^{4}$ Universidad Autónoma de Nayarit, Secretaría de Investigación y Posgrado, Unidad de Tecnología de Alimentos. Ciudad de la Cultura Amado Nervo. Boulevard Tepic-Xalisco s/n. 63155 Tepic, Nayarit, México.

${ }^{5}$ Universidad Autónoma Chapingo, Departamento de Fitotecnia. Carretera México-Texcoco km 38.5, Chapingo. 56230 Texcoco, Estado de México, México.
}

\section{RESUMEN}

El biocarbón es un material rico en carbono, obtenido por conversión termoquímica elaborado de materiales orgánicos en un ambiente limitado de oxígeno, que puede usarse como mejorador de suelos. El objetivo de la investigación fue evaluar el rendimiento de biocarbón convertido, la concentración nutrimental y la estructura del biocarbón de ápices de caña de azúcar elaborado mediante carbonización hidrotérmica y adición de catalizadores orgánicos. El diseño experimental fue completamente al azar con seis repeticiones. Los tratamientos fueron tres catalizadores: ácido cítrico, maleico y propiónico en concentraciones al 5 y $10 \%$ cada uno, los cuales se adicionaron en la elaboración del biocarbión; el testigo fue biocarbón sin adición de catalizador, para un total de siete tratamientos. Se determinó el rendimiento de conversión de biomasa a biocarbón, la concentración nutrimental total de $\mathrm{N}, \mathrm{P}, \mathrm{Ca}, \mathrm{Mg}$ y el elemento $\mathrm{Na}$; también se determinó la concentración extraíble de los mismos nutrimentos con excepción del $\mathrm{Na}$, así como el $\mathrm{pH}$ y la conductividad eléctrica y la estructura del biocarbón. Los mayores rendimientos del biocarbón elaborado con ápices de caña de azúcar mediante carbonización hidrotérmica fueron de $37.8 \%$ con la adición de ácido cítrico a $10 \%$ como catalizador, así como 34.9 y $36.1 \%$ con la adición de ácido maleico a 5 y $10 \%$, respectivamente. En general, por la concentración nutrimental total y extraíble del biocarbón, su conductividad eléctrica (0.30-0.46 dS m $\left.\mathrm{m}^{-1}\right)$, así como su estructura con microesferas, el biocarbón de ápices de caña de azúcar podría servir como mejorador de suelos, especialmente en suelos alcalinos debido al $\mathrm{pH}$ ácido del biocarbón producido.

Palabras clave: carbonización hidrotérmica, catalizadores orgánicos, concentración nutrimental, mejorador de suelos, Saccharum officinarum.

\section{SUMMARY}

Biochar is a carbon-rich material obtained by thermochemical conversion produced from organic materials in an oxygen-limited environment, which can be used as a soil enhancer. The objective of the research

Cita recomendada:

Pérez-Cabrera, C. A., Juárez-López, P., Anzaldo-Hernández, J., Alia-Tejacal, I., Salcedo-Pérez, E., Guillén-Sánchez, D., Balois-Morales, R., López-Martínez, V. y Castro-Brindis, R. (2021). Caracterización química de biocarbón de ápices de caña de azúcar elaborado mediante carbonización hidrotérmica y adición de catalizadores orgánicos. Terra Latinoamericana, 39, 1-11. e936. https://doi.org/10.28940/terra.v39i0.936 
was to evaluate the yield of converted biochar, nutrient concentration and structure of sugarcane tips biochar produced by hydrothermal carbonization and addition of organic catalysts. The experimental design was completely randomized with six replicates. Treatments were three catalysts: citric, maleic and propionic acid in 5 and $10 \%$ concentrations each, which were added during biochar production. The respective control was biochar without addition of any catalyst, for a total of seven treatments. The biomass to biochar conversion yield and the total nutrient concentration of $\mathrm{N}, \mathrm{P}, \mathrm{Ca}, \mathrm{Mg}$ and $\mathrm{Na}$ were determined. The extractable concentration of the same nutrients except for $\mathrm{Na}$ was also determined, as well as the $\mathrm{pH}$ and the electrical conductivity and structure of the biochar. The highest yields of biochar made from sugarcane tips by hydrothermal carbonization were $37.8 \%$ with the addition of citric acid at $10 \%$ as catalyst, as well as 34.9 and $36.1 \%$ with the addition of maleic acid at 5 and $10 \%$, respectively. In general, due to the total and extractable nutrient concentration of biochar, its electrical conductivity $\left(0.30-0.46 \mathrm{dS} \mathrm{m}^{-1}\right)$, as well as its microsphere structure, sugarcane tips biochar could act as a soil enhancer, especially in alkaline soils due to the acid $\mathrm{pH}$ of the biochar produced.

Index words: hydrothermal carbonization, organic catalysts, nutrient concentration, soil improver, Saccharum officinarum.

\section{INTRODUCCIÓN}

El biocarbón es un material sólido rico en carbono, que se obtiene por la conversión termoquímica de materiales orgánicos en un ambiente limitado o carente de oxígeno (Zheng, Holm y Spokas, 2016), el cual tiene propiedades físicas y químicas aptas para el almacenamiento de carbono a largo plazo en un medio natural $\mathrm{y}$, potencialmente, mejora de la fertilidad de los suelos (Ibarrola, Evar y Reay, 2013).

El biocarbón posee estabilidad y por ello, su aplicación al suelo como mejorador presenta dos ventajas principales: la primera, es por el secuestro del carbono, ya que el suelo actúa como un almacén o reservorio, reduciendo de este modo las emisiones directas de carbono a la atmósfera (Steiner, 2016); la segunda ventaja es que mejora la capacidad del suelo para retener la humedad, la cual se debe a su morfología altamente porosa, aumento en la capacidad de intercambio catiónico (Gallo-Saravia, Lugo y Barrera, 2018; Adeyemi y Idowu, 2017), así como liberación lenta de nutrimentos (Ibarrola et al., 2013).

Existen varias técnicas termoquímicas para la producción de biocarbón, tales como pirolisis (lenta, rápida, y ultrarrápida), gasificación, y carbonización hidrotérmica (HTC, por sus siglas en inglés) (Adeyemi y Idowu, 2017; Zheng et al., 2016). Las técnicas de pirolisis y gasificación requieren que la materia prima (biomasa o residuos) esté seca previo al proceso de carbonización; sin embargo, esta etapa se puede omitir con la técnica de carbonización hidrotérmica, la cual es una ventaja para el aprovechamiento de residuos con elevado contenido de agua o residuos de cosechas recién cortados (Quesada-Kimzey, 2012).

En México las investigaciones de biocarbón con fines agrícolas son incipientes; sin embargo, existe interés creciente en la elaboración de biocarbón para el aprovechamiento de residuos de cosecha y residuos industriales. Orozco-Gutiérrez y LiraFuentes (2020) reportaron rendimiento de $27 \%$ en biocarbón elaborado a $550{ }^{\circ} \mathrm{C}$ a partir de ramas de bambú Guadua angustifolia. Velázquez-Machuca et al. (2019) concluyeron que el biocarbón de lodos residuales puede usarse como mejorador de suelos agrícolas por su alto contenido nutrimental y su bajo contenido de metales tóxicos. Medina y Medina (2018) construyeron y evaluaron un equipo de pirólisis lenta, autotérmico y móvil con capacidad para procesar de 300 a 400 kg de biomasa; con el equipo mencionado elaboraron biocarbón a partir de ramas podadas de aguacate, con un rendimiento de $16 \%$ de biocarbón, $\mathrm{pH}$ alcalino (10.25) y alta capacidad de intercambio catiónico (46 cmolc $\mathrm{kg}^{-1}$ ). Mientras que, Concilco, Moreno, García, Quiroga y García (2018) al evaluar un biocarbón comercial de bambú en el cultivo de avena forrajera, reportaron que la aplicación de $25 \mathrm{Mg} \mathrm{ha}^{-1} \mathrm{de}$ biocarbón con fertilización química 120-60-00 (NPK) aumentó $34 \%$ la altura de planta y $103 \%$ la materia fresca en comparación con el tratamiento testigo.

Por otra parte, Wang y Wang (2019) mencionan que la función de la adición de ácidos en la elaboración de biocarbón es eliminar impurezas, metales pesados e introducir grupos carboxilos en la superficie del biocarbón para mejorar su estructura; asimismo. Se ha reportado que la adición de ácidos como catalizadores en el proceso de su elaboración puede 
mejorar las propiedades físicas y químicas, como se ha observado en biocarbón elaborado con residuos agroindustriales mediante la técnica de carbonización hidrotérmica (Chaparro-Garnica, Mostazo, Salinas, Morallon y Cazorla, 2020; Silva et al., 2017). Velázquez-Maldonado et al. (2019) con la adición de catalizadores orgánicos en la elaboración de biocarbón de cascarilla de arroz, obtuvieron incremento en el rendimiento de conversión de la biomasa inicial, así como mayor materia orgánica y concentración nutrimental. Estos mismos autores concluyeron que por sus características de rendimiento, materia orgánica y concentración nutrimental, el biocarbón de cascarilla de arroz puede utilizarse como mejorador de suelo; sin embargo, también indican que es necesario realizar más estudios para conocer las características químicas de biocarbones elaborados con otros materiales vegetales.

Generalmente, la estructura del biocarbón es amorfa, constituida por partículas de diferentes tamaños, lo que depende de la materia prima, de la de técnica y el tiempo en su elaboración (EscalanteRebolledo et al., 2016). En este sentido, Sevilla y Titirici (2012) mencionan que la temperatura de conversión influye en el diámetro y en la distribución del tamaño de las partículas. La estructura porosa del biocarbón puede ser la responsable de la elevada capacidad de retención de agua; además, es un hábitat idóneo para la proliferación de microorganismos que mejoran el suelo (Stadler-Kaulich y Perteguer, 2018).

La elaboración de biocarbón es una técnica que se puede implementar donde hay suficiente biomasa disponible para ser usados como materia prima y que estos materiales no compitan con otros usos, principalmente si se generan productos de mayor valor económico (Escalante-Rebolledo et al., 2016).

Por otro lado, en México, el cultivo de caña de azúcar se siembra en 16 entidades federativas. En el 2019, se reportó una superficie cosechada de 781567.87 ha, con producción de $58894343.71 \mathrm{Mg}$ (SIAP, 2020). Aunque la cantidad de residuos orgánicos que genera el cultivo de la caña de azúcar depende de diversos factores como variedad de la caña, niveles de productividad, fechas de cosecha y eficiencia de la cosechadora en el corte, así como la recolección y limpieza (Ortiz-Laurel, Salgado, Castelán y Córdova, 2012); en general, el cultivo de la caña de azúcar produce residuos (punta o cogollo) entre 35 y $60 \mathrm{Mg} \mathrm{ha}^{-1}$ (GuerreroPeña, Gómez, Hernández, Salinas y Velasco, 2017), los cuales se pueden aprovechar en la producción de biocarbón. Por lo anteriormente expuesto, el objetivo del presente estudio fue evaluar el rendimiento de biocarbón convertido, la concentración nutrimental y la estructura del biocarbón de ápices de caña de azúcar elaborado con carbonización hidrotérmica y adición de catalizadores orgánicos.

\section{MATERIALES Y MÉTODOS}

\section{Material Vegetal, Tratamientos y Elaboración de Biocarbón}

Los ápices de caña de azúcar (ACa) se cosecharon el 15 junio de 2018 en Zapopan, Jalisco. El biocarbón se elaboró del 16 al 25 del mismo mes y las variables se evaluaron durante julio y agosto del mismo año. El biocarbón se elaboró mediante la técnica de carbonización hidrotérmica (HTC, por sus siglas en inglés) descrita por Velázquez-Maldonado et al. (2019). Los ACa se cortaron en trozos de $5 \mathrm{~cm}$, se pesaron $200 \mathrm{~g}$ los cuales contenían humedad de $53.73 \%$ y se les adicionó agua destilada $(88.54 \mathrm{~mL})$ para obtener una humedad final de $100 \%$. Los tratamientos consistieron en la adición de ácido cítrico, maleico y propiónico como catalizadores en dos concentraciones cada uno (5 y 10\%); el tratamiento testigo fue biocarbón al que se adicionó agua destilada (sin catalizador), para un total de siete tratamientos. Posteriormente, las muestras se colocaron en un reactor modelo JAYME marca Deutsch and Neumann ${ }^{\circledR}$, durante $19 \mathrm{~h}$ a $200^{\circ} \mathrm{C}$; después del tiempo transcurrido, se dejó enfriar el reactor a temperatura ambiente. Al material obtenido se le realizó un triple lavado (agua destilada-etanol $96 \%$-agua destilada) y filtrado. Por último, el biocarbón se secó en una estufa de aire circulante (modelo F210 marca Felisa ${ }^{\circledR}$ ) a $70{ }^{\circ} \mathrm{C}$ durante 12 h. En la Figura 1 se muestra el material vegetal de ápices de caña de azúcar antes y después de la carbonización hidrotérmica.

\section{Rendimiento de Biocarbón}

El rendimiento del biocarbón se determinó mediante la diferencia entre el peso de los ápices de caña de azúcar (biomasa inicial) y el peso de los ápices de caña de azúcar convertidos en biocarbón; posteriormente, los valores obtenidos se expresaron en porcentaje. 

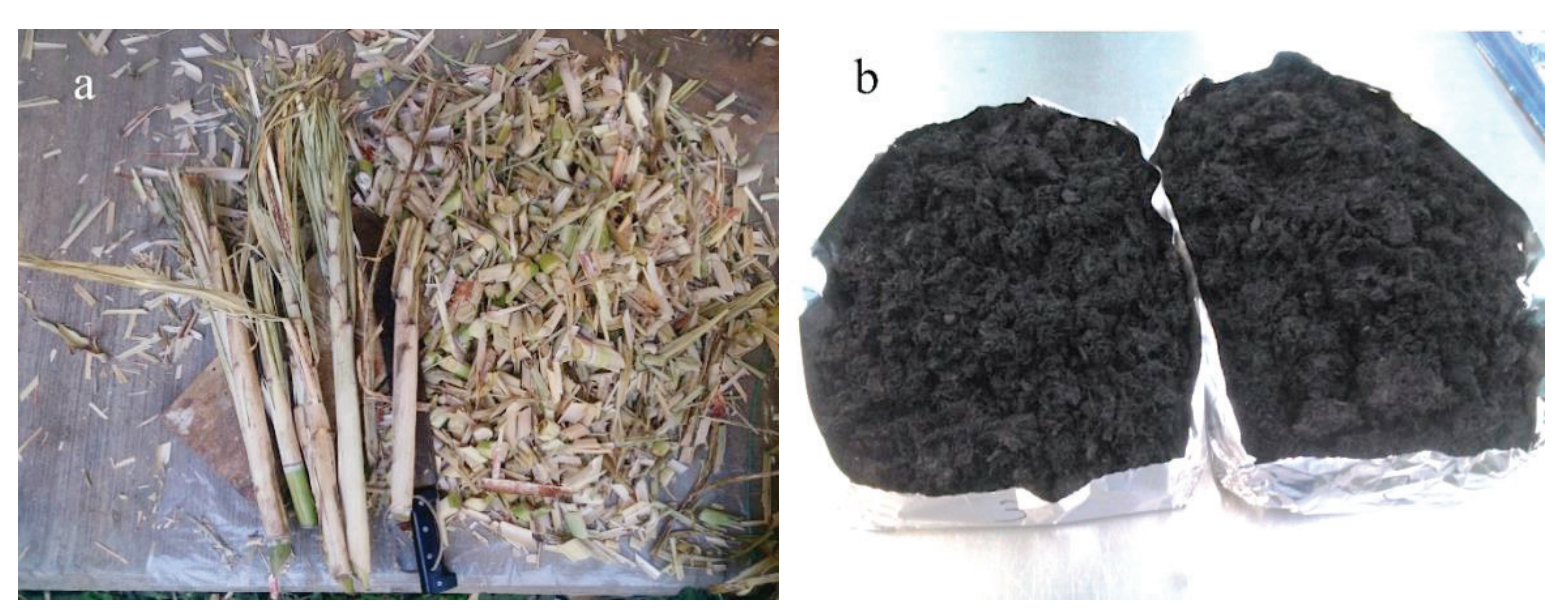

Figura 1. a) Biomasa de ápices de caña de azúcar. b) Biocarbón de caña de azúcar elaborado mediante carbonización hidrotérmica.

Figure 1. a) Biomass of sugarcane apices. b) Biochar from sugar cane made by hydrothermal carbonization.

\section{Concentración Nutrimental}

La concentración total de $\mathrm{N}$ se determinó por el método Kjedahl, $\mathrm{P}$ total por el método de molibdato de sodio con p-metilaminofenol sulfato en un espectrofotómetro ultravioleta visible modelo SQUV2800, marca UNICO ${ }^{\circledR}$; los contenidos totales de K, $\mathrm{Ca}, \mathrm{Mg}$ y $\mathrm{Na}$ por digestión ácida y espectrofotometría de absorción atómica modelo 932 Plus, marca GBC $^{\circledR}$ (Cottenie, 1994); asimismo, el análisis de elementos extraíbles de $\mathrm{P}, \mathrm{K}, \mathrm{Ca}$ y $\mathrm{Mg}$ se realizó según las metodologías descritas por la Iniciativa Internacional del Biocarbón (IBI, 2015). También se determinó el $\mathrm{pH}$ en agua con relación 1:20 por potenciometría y la conductividad eléctrica en relación 1:20 con un conductímetro en el mismo recipiente usado para medir pH (IBI, 2015).

\section{Estructura de Biocarbones}

La estructura de los biocarbones se analizó por microscopía electrónica de barrido (SEM, por sus siglas en inglés) con un microscopio modelo MIRA 3 LMU, marca TESCAN ${ }^{\circledR}$. Se molió 0.01 g de biocarbón en morteros ágata y se colocó en un portaobjetos con cinta adhesiva doble cara, sobre la cinta adhesiva se colocó una capa fina de las muestras. Posteriormente, a las muestras adheridas se les agregó con oro con un dispositivo de pulverización catódica modelo SPIMODULE Sputter Coater, marca SPI SUPPLIES ${ }^{\circledR}$, según metodología descrita por Varela, Rivera, Huang, Chien y Wang (2013).

\section{Diseño Experimental y Análisis Estadístico}

El diseño experimental fue completamente al azar con seis repeticiones. Para asegurar la normalidad, los datos expresados en porcentaje se transformaron con la raíz cuadrada del arcoseno. Los resultados se sometieron a un análisis de varianza y prueba de medias de Tukey $(P \leq 0.05)$ mediante el programa estadístico SAS versión 9.1.3 (SAS, 2004).

\section{RESULTADOS Y DISCUSIÓN}

\section{Rendimiento de Biocarbón}

La adición de ácidos orgánicos como catalizadores en la elaboración de biocarbón de ápices de caña de azúcar mediante carbonización hidrotérmica (HTC), influyó en el rendimiento del biocarbón convertido en comparación con el testigo (Cuadro 1). Hubo diferencias estadísticas entre los tratamientos $(P \leq 0.05)$. La adición de ácido cítrico al 10\%, y el ácido maleico al 5 y $10 \%$ fueron estadísticamente iguales. Los ácidos orgánicos anteriormente mencionados presentaron los mayores valores en rendimiento con 37.8, $34.9 \mathrm{y}$ $36.1 \%$, respectivamente; esto representa incremento en conversión de la biomasa a biocarbón de 8.2, 5.3 y 
$6.5 \%$ respectivamente, comparado con el tratamiento testigo. Velázquez-Maldonado et al. (2019) reportaron rendimiento de $66 \%$ con la adición de ácido maleico y ácido cítrico al 10\%, en la elaboración de biocarbón de cascarilla de arroz mediante HTC. Al respecto, Lynam, Toufic, Vasquez y Coronella (2012) mencionan que el rendimiento del biocarbón se ve influenciado por el uso de catalizadores durante el proceso de carbonización hidrotérmica; es decir, favorece el proceso de hidrólisis de la celulosa y hemicelulosa de los materiales vegetales. Durante el proceso de carbonización hidrotérmica se producen reacciones de hidrólisis, deshidratación, descarboxilación, aromatización, policondensación y polimerización para producir diversos materiales carbonosos, como esferas de carbono y nano fibras de carbono (Zhang et al., 2020). Además, la adición de catalizadores proporciona una funcionalización química en la superficie del biocarbón (Xu, Xia, Jiang, Li y Xue, 2018; Román, Nabais, Laginhas, Ledesma y González, 2012).

Singh, Singh y Purakayastha (2018) reportaron rendimiento de $52.5 \%$ en la conversión de la materia prima utilizando bagazo de caña de azúcar mediante la técnica de pirólisis a $400{ }^{\circ} \mathrm{C}$. Silva et al. (2017) en la elaboración de biocarbón con mezcla de vinaza y bagazo de caña de azúcar mediante HTC, encontraron rendimiento entre 17.6 a $46.5 \%$ en función de la temperatura y el tipo de catalizador empleado (ácido sulfúrico, fosfórico, bórico, hidróxido de sodio, hidróxido de potasio, cloruro ferroso tetrahidratado $\mathrm{y}$ sulfato de amonio); el mayor rendimiento del biocarbón se obtuvo con temperatura de $150{ }^{\circ} \mathrm{C}$ con ácido sulfúrico, fosfórico e hidróxido de sodio como catalizadores. Recientemente, Orozco-Gutiérrez y Lira-Fuentes (2020) reportaron que el rendimiento del biocarbón aumentó de 10 a $27 \%$ con temperaturas de 350 a $550{ }^{\circ} \mathrm{C}$, mientras que temperatura por encima de $550{ }^{\circ} \mathrm{C}$ disminuyó el rendimiento del biocarbón elaborado de bambú (Guadua angustifolia) mediante pirólisis lenta.

En adición a lo anterior, Schlegel, Ibrahim, Kipping, Ortiz y Fras (2018) menciona que un factor importante a considerar en el rendimiento del biocarbón es la composición de la materia prima, debido a que se relaciona directamente con la eficiencia de conversión y calidad; es decir, para que la eficiencia de conversión de materia prima a biocarbón sea alta, el material vegetal debe ser rico en lignina respecto a la celulosa. Asimismo, se ha reportado que los parámetros de temperatura, tiempo de reacción, $\mathrm{pH}$ del medio de reacción y la adición de catalizadores influyen sobre el rendimiento y propiedades físicas y químicas del biocarbón (Silva et al., 2017; Escalante-Rebolledo et al., 2016).

Cuadro 1. Rendimiento, concentración total de macronutrimentos y de sodio (Na) en biocarbón de ápices de caña de azúcar con tres catalizadores.

Table 1. Yield, total concentration of macronutrients and sodium (Na) in biochar from sugarcane tips with three catalysts.

\begin{tabular}{|c|c|c|c|c|c|c|c|}
\hline Tratamientos & Rendimiento & $\mathrm{N}$ & $\mathrm{P}$ & $\mathrm{K}$ & $\mathrm{Ca}$ & $\mathrm{Mg}$ & $\mathrm{Na}$ \\
\hline & \multicolumn{7}{|c|}{ 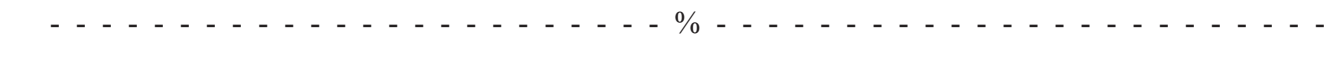 } \\
\hline Testigo ( $\sin$ catalizador) & $29.6 \mathrm{~cd}^{\dagger}$ & $1.789 \mathrm{a}$ & $0.121 \mathrm{~b}$ & $0.240 \mathrm{~cd}$ & $0.029 \mathrm{a}$ & $0.178 \mathrm{ab}$ & $0.232 \mathrm{ab}$ \\
\hline Ácido cítrico 5\% & $32.2 \mathrm{bc}$ & $1.446 \mathrm{~b}$ & $0.162 \mathrm{a}$ & $0.185 \mathrm{~d}$ & $0.018 \mathrm{ab}$ & $0.087 \mathrm{bc}$ & $0.246 \mathrm{ab}$ \\
\hline Ácido cítrico $10 \%$ & $37.8 \mathrm{a}$ & $1.103 \mathrm{c}$ & $0.109 \mathrm{~b}$ & $0.419 \mathrm{a}$ & $0.018 \mathrm{ab}$ & $0.257 \mathrm{a}$ & $0.251 \mathrm{a}$ \\
\hline Ácido maleico 5\% & $34.9 \mathrm{ab}$ & $1.299 \mathrm{bc}$ & $0.123 \mathrm{~b}$ & $0.325 \mathrm{bc}$ & $0.024 \mathrm{ab}$ & $0.105 \mathrm{bc}$ & $0.241 \mathrm{ab}$ \\
\hline Ácido maleico $10 \%$ & $36.1 \mathrm{ab}$ & $1.372 \mathrm{bc}$ & 0.169 a & $0.340 \mathrm{ab}$ & $0.029 \mathrm{a}$ & $0.055 \mathrm{c}$ & $0.243 \mathrm{ab}$ \\
\hline Ácido propiónico 5\% & $27.3 \mathrm{~d}$ & $1.201 \mathrm{bc}$ & $0.132 \mathrm{~b}$ & $0.185 \mathrm{~d}$ & $0.013 \mathrm{~b}$ & $0.176 \mathrm{ab}$ & $0.246 \mathrm{ab}$ \\
\hline Ácido propiónico 10\% & $29.6 \mathrm{~cd}$ & $1.421 \mathrm{~b}$ & $0.163 \mathrm{a}$ & $0.334 \mathrm{ab}$ & $0.028 \mathrm{a}$ & $0.237 \mathrm{a}$ & $0.226 \mathrm{~b}$ \\
\hline DMS & 3.9 & 0.289 & 0.029 & 0.092 & 0.012 & 0.118 & 0.023 \\
\hline
\end{tabular}

Letras distintas en la misma columna indican diferencias significativas, según la prueba de Tukey $(P \leq 0.05)$. DMS $=$ diferencia mínima significativa.

${ }^{\dagger}$ Different letters in the same column indicate significant differences, according to Tukey's test $(P \leq 0.05)$. DMS $=$ least significant difference. 


\section{Concentración Total de Nutrimentos}

Se encontraron diferencias significativas entre los tratamientos $(P \leq 0.05)$. Con excepción del $\mathrm{N}$, al menos una concentración de catalizador presentó valores similares o mayores en la concentración nutrimental (Cuadro 1). Estos resultados concuerdan con lo reportado por Velázquez-Maldonado et al. (2019) quienes mencionan que la adición de catalizadores orgánicos favoreció la concentración de macronutrimentos en biocarbón de cascarilla de arroz. Por otra parte, Silva et al. (2017) reportaron que en biocarbón mezcla de vinaza y bagazo de caña de azúcar, el $\mathrm{N}$ aumentó al utilizar $\mathrm{H}_{3} \mathrm{BO}_{3}$ como catalizador en el medio de reacción; sin embargo, al usar $\left(\mathrm{NH}_{4}\right)_{2} \mathrm{SO}_{4}$ incrementó la concentración de $\mathrm{N}, \mathrm{K}$ y Ca, la adición de $\mathrm{H}_{2} \mathrm{SO}_{4}$ aumentó solo el $\mathrm{K}$, mientras que al agregar $\mathrm{H}_{3} \mathrm{PO}_{4}$ se incrementaron las concentraciones de $\mathrm{K}, \mathrm{Mg}$, Ca y $\mathrm{P}$, en tanto que el $\mathrm{Mg}$ aumentó al utilizar $\mathrm{NaOH}$ y $\mathrm{KOH}$ como catalizadores.

Con excepción de la concentración de $\mathrm{Ca}$ que varió de 0.18 a $15.8 \%$, los valores encontrados en el presente estudio se encuentran dentro de los intervalos de concentración nutrimental reportados por Cho et al. (2017) quienes en cinco biocarbones elaborados a partir de astillas de pino, viruta de roble, piña de pino, cascarilla de arroz y caparazón de cangrejo, encontraron concentraciones en porcentaje: $\mathrm{N}$ (0.07-3.63),
P (0.026-1.21), K (0.097-0.645), Mg (0.075-1.04) у $\mathrm{Na}(0.035-0.734)$. Por otro lado, los valores de N y P del presente estudio son menores a los reportados por Bento et al. (2019) quienes encontraron $3.42 \%$ para $\mathrm{N}$ y $4.71 \%$ para $\mathrm{P}$ en biocarbón elaborado de mezcla de vinaza y bagazo de caña de azúcar mediante la técnica de HTC con ácido fosfórico como catalizador. Es decir, la composición de la materia prima, técnica de conversión, temperatura, entre otros factores influyen sobre las características químicas del biocarbón. En cuanto al elemento $\mathrm{Na}$, los valores obtenidos (0.226-0.251\%) se encuentran dentro del intervalo indicado por Alcántar-González y Trejo-Téllez (2012) quienes mencionan que el $\mathrm{Na}$ total puede encontrarse en niveles entre 0.1 y $1 \%$.

\section{pH y Conductividad Eléctrica}

En cuanto a $\mathrm{pH}$ y conductividad eléctrica (CE) hubo diferencias estadísticas $(P \leq 0.05)$, (Cuadro 2$)$. El pH obtenido varió de 4.46 (ácido cítrico 10\%) a 6.75 (ácido propiónico 5\%). La CE de los biocarbones varió de $0.30 \mathrm{dS} \mathrm{m}^{-1}$ (ácido cítrico al $5 \%$ ) a $0.46 \mathrm{dS} \mathrm{m}^{-1}$ (ácido maleico al 10\%). Estos dos parámetros son de importancia ya que $\mathrm{pH}$ y $\mathrm{CE}$ se utilizan como indicadores de disponibilidad de nutrimentos y concentración de sales, cuando son aplicados al suelo. Los resultados obtenidos son aproximados a los reportados por Silva

Cuadro 2. pH, CE y concentración nutrimental extraíble en biocarbón de ápices de caña de azúcar con tres catalizadores.

Table 2. pH, EC and extractable nutrient concentration in biochar from sugarcane tips with three catalysts.

\begin{tabular}{|c|c|c|c|c|c|c|}
\hline & & $\mathrm{dS} \mathrm{m} \mathrm{m}^{-1}$ & \multicolumn{4}{|c|}{$\ldots \ldots \ldots-\ldots \mathrm{mg} \mathrm{kg}^{-1}$} \\
\hline Ácido cítrico 5\% & $5.22 \mathrm{c}$ & $0.30 \mathrm{~cd}$ & $128.88 \mathrm{ab}$ & $652.36 \mathrm{c}$ & $80.07 \mathrm{~b}$ & $576.64 \mathrm{a}$ \\
\hline Ácido maleico 5\% & $4.71 \mathrm{~d}$ & $0.40 \mathrm{ab}$ & $150.85 \mathrm{a}$ & $958.28 \mathrm{~b}$ & $79.24 \mathrm{~b}$ & $562.37 \mathrm{a}$ \\
\hline Ácido maleico $10 \%$ & $4.60 \mathrm{~d}$ & $0.46 \mathrm{a}$ & $136.26 \mathrm{a}$ & $724.14 \mathrm{c}$ & $79.24 \mathrm{~b}$ & $258.75 \mathrm{~b}$ \\
\hline Ácido propiónico 5\% & $6.75 \mathrm{a}$ & $0.33 \mathrm{bc}$ & $97.78 \mathrm{bc}$ & $702.86 \mathrm{c}$ & $124.61 \mathrm{a}$ & $437.07 \mathrm{a}$ \\
\hline Ácido propiónico $10 \%$ & $6.20 \mathrm{~b}$ & $0.32 \mathrm{bc}$ & $60.83 \mathrm{~d}$ & $546.76 \mathrm{c}$ & $79.24 b$ & $176.83 \mathrm{~b}$ \\
\hline
\end{tabular}

Letras distintas en la misma columna indican diferencias significativas, según la prueba de Tukey $(P \leq 0.05)$. DMS $=$ diferencia mínima significativa.

$\dagger$ Different letters in the same column indicate significant differences, according to Tukey’s test $(P \leq 0.05)$. DMS $=1$ least significant difference. 
et al. (2017) en biocarbón mezcla de vinaza y bagazo de caña de azúcar utilizando HTC, quienes encontraron que, con y sin catalizadores el $\mathrm{pH}$ varió entre $3.95 \mathrm{a}$ 5.05. Por su parte, Bento et al. (2019) reportaron que el valor de $\mathrm{pH}$ de 3.54 en biocarbón mezcla de vinaza y bagazo de caña de azúcar elaborados con $\mathrm{HTC}$ a $230^{\circ} \mathrm{C}$ con ácido fosfórico como catalizador puede deberse a la presencia de grupos carboxílicos en la estructura de los biocarbones.

Velázquez-Machuca et al. (2019) reportaron $\mathrm{pH}$ cercanos a neutro (6.33 a 7.33) y CE entre $0.522 \mathrm{dS} \mathrm{m}^{-1}$ a $3.11 \mathrm{dS} \mathrm{m}^{-1}$ en biocarbón de lodos residuales elaborados mediante la técnica de pirolisis a temperaturas de $280-330{ }^{\circ} \mathrm{C}$. Mientras Medina y Medina (2018) reportaron en biocarbón de esquilmos de aguacate (Persea americana) elaborado con la técnica de pirolisis lenta con temperaturas entre 360 a $700{ }^{\circ} \mathrm{C}$, valores de $\mathrm{pH}$ alcalinos (10.25) y bajo contenido de sales $0.92 \mathrm{dS} \mathrm{m}^{-1}$. De igual forma, Concilco et al. (2018) en biocarbón comercial de biomasa de bambú (Bambusa oldhammi) elaborado por pirólisis a $650{ }^{\circ} \mathrm{C}$, reportaron $\mathrm{pH}$ de 8.85 y CE de $2.55 \mathrm{dS} \mathrm{m}^{-1}$. Además, Cho et al. (2017) en biocarbones elaborados a partir de astillas de pino, astillas de roble, piña de pino, cascarilla de arroz y caparazón de cangrejo a temperaturas entre 200 a $250{ }^{\circ} \mathrm{C}$, reportaron valores de $\mathrm{pH}$ entre 5.1 a 8.8 y CE entre 0.005 a $1.24 \mathrm{dS} \mathrm{m}^{-1}$, en dependencia de la materia prima utilizada.

Los valores de $\mathrm{pH}$ de la mayoría de los biocarbones del presente estudio, elaborados con la técnica de HTC, indican que son ácidos por lo que pueden ser una alternativa para utilizarse como enmiendas en suelos o sustratos alcalinos, mientras que los biocarbones con $\mathrm{pH}$ altos pueden potencialmente sustituir al encalado agrícola que se recomienda para enmendar suelos ácidos.

\section{Concentración Extraíble de Nutrimentos}

Se obtuvieron diferencias significativas $(P \leq 0.05)$ entre los tratamientos, según el catalizador empleado (Cuadro 2). Al igual que en concentración total de macronutrimentos, al menos una concentración de los catalizadores tuvo valores similares o superiores al testigo; al respecto, no se encontraron reportes en la literatura revisada respecto a la concentración nutrimental extraíble en biocarbones asociada a los catalizadores utilizados en el presente estudio.
Sin embargo, Bento et al. (2019) reportan concentraciones nutrimentales extraíbles de 2.82\% de $\mathrm{K}, 2.21 \%$ de $\mathrm{Ca}, 2.67 \%$ de $\mathrm{Mg}$ en biocarbones elaborados con mezcla de vinaza y bagazo de caña de azúcar.

Los resultados del presente estudio indican, que el uso de ácidos orgánicos utilizados favoreció la concentración de algunos macronutrimentos totales y extraíbles en el biocarbón de ápices de caña de azúcar, ya que la mayoría de los minerales fueron superiores al testigo (sin catalizador). Silva et al. (2017) concluyeron que los catalizadores influyen en la inmovilización de los macronutrimentos del biocarbón y la naturaleza química de cada catalizador puede repercutir en la concentración nutrimental; asimismo, mencionan que la inmovilización de los macros y micronutrimentos ocurre por adsorción en la superficie del biocarbón, así como también por la precipitación de compuestos insolubles. Bento et al. (2019) indican que la presencia de grupos funcionales como ácidos carboxílicos en la estructura química del biocarbón, puede proporcionar mayor capacidad de absorción de cationes metálicos como el $\mathrm{Ca}, \mathrm{Mg}$ y $\mathrm{Fe}$. La cantidad de grupos hidroxilo y carboxilo, en la superficie del biocarbón, depende del tipo ácido empleado como catalizador en el medio de reacción, por ejemplo, el ácido cítrico aporta tres grupos carboxilos, mientras que el ácido maleico y propiónico aportan dos y un grupo carboxílicos, respectivamente.

Es necesario realizar más investigaciones sobre las características químicas de biocarbones para utilizarse como enmienda de suelos, sobre todo cuando se usan nuevos materiales vegetales, ya que las propiedades del biocarbón son afectadas por la composición de la materia prima, técnicas termoquímicas empleadas en su elaboración, intervalo de calentamiento, temperatura, presión del reactor y uso de catalizadores (EscalanteRebolledo et al., 2016)

\section{Estructura del Biocarbón}

En las micrografías se observaron microesferas de carbón con los tres catalizadores empleados y el testigo (Figura 2). Los valores promedio del diámetro de las microesferas fueron: testigo ( $\sin$ catalizador), $0.706 \mu \mathrm{m}$; ácido cítrico (5\%), $1.333 \mu \mathrm{m}$; ácido cítrico (10\%), $0.329 \mu \mathrm{m}$; ácido maleico $(5 \%), 1.799 \mu \mathrm{m}$; ácido maleico $(10 \%), 2.354 \mu \mathrm{m}$; ácido propiónico, (5\%), $0.708 \mu \mathrm{m}$; y ácido propiónico (10\%), $0.999 \mu \mathrm{m}$. 


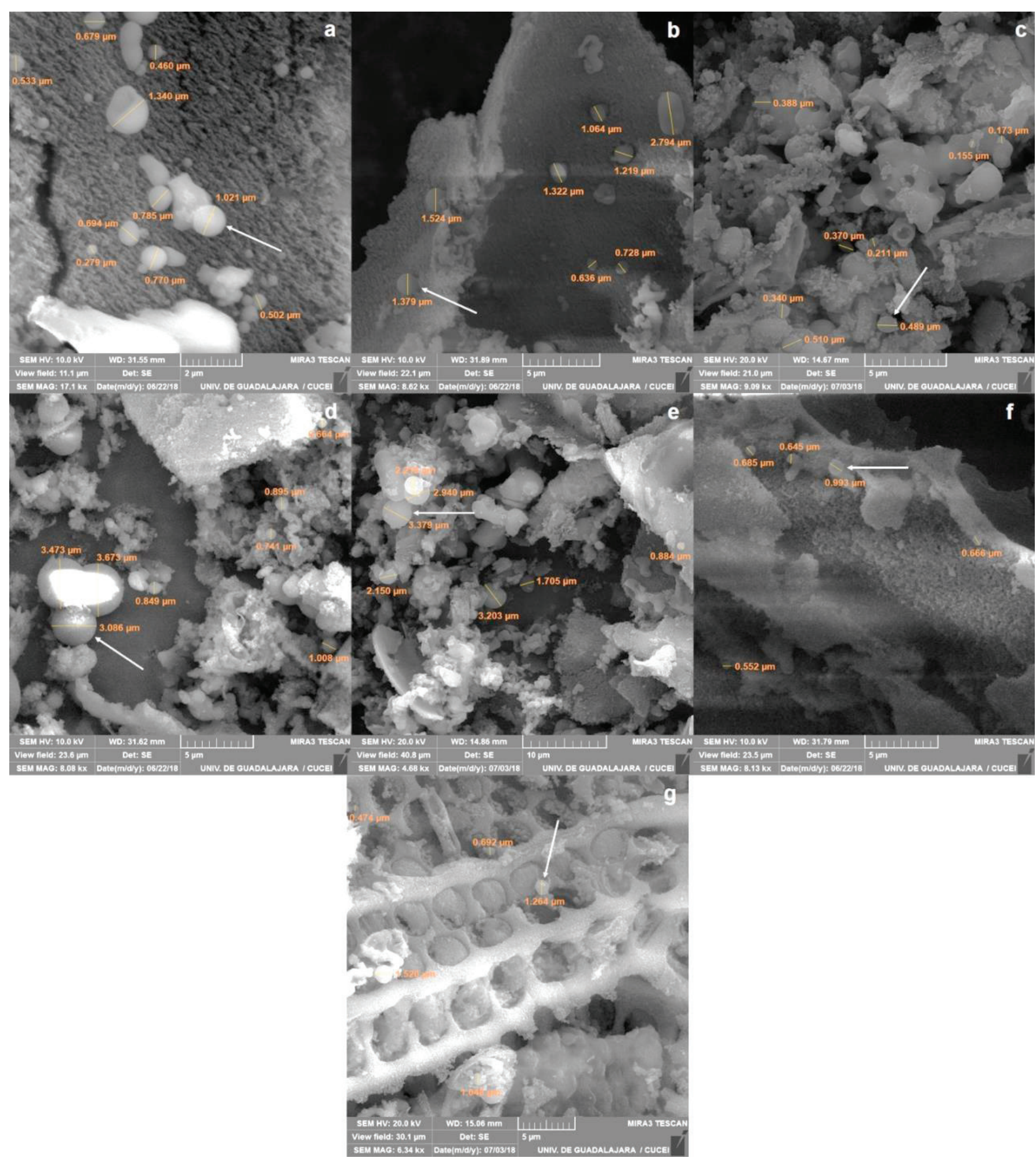

Figura 2. Microesferas en el biocarbón de ápices de caña de azúcar con tres catalizadores. a) testigo (sin catalizador), b) ácido cítrico al $5 \%$, c) ácido cítrico al $10 \%$, d) ácido maleico al 5\%, e) ácido maleico al $10 \%$, f) ácido propiónico al $5 \%$ y g) ácido propiónico al $10 \%$. Las fechas señalan la presencia de microesferas de carbón en cada micrografía.

Figure 2. Microspheres in sugarcane apex biochar with three catalysts. a) control (without catalyst), b) $5 \%$ citric acid, c) $10 \%$ citric acid, d) 5\% maleic acid, e) $10 \%$ maleic acid, f) $5 \%$ propionic acid and g) $10 \%$ acid propionic. Dates indicate the presence of carbon microspheres in each micrograph. 
El diámetro de las microesferas del presente estudio es mayor a los reportados por Velázquez-Maldonado et al. (2019) quienes en biocarbón de cascarilla de arroz obtuvieron valores de 0.055 a $0.084 \mu \mathrm{m}$, estos resultados podrían deberse a la composición de la materia prima de la cascarilla de arroz, la cual es rica en lignina en comparación a la celulosa que contienen los ápices de caña. Estos mismos autores, mencionan que los biocarbones elaborados con la técnica HTC generalmente son de $\mathrm{pH}$ ácidos, con superficie porosa, con mejor capacidad de adsorción al incrementar su superficie de área. Blanco-Canqui (2017) señala que las partículas del biocarbón pueden mezclarse con el suelo y formar agregados más grandes, lo cual mejora su estructura.

En este contexto, el aumento de la concentración de ácido maleico y propiónico al $10 \%$ como catalizadores, influyó en el tamaño de las microesferas de carbón, debido a que hubo tendencia de incremento de tamaño comparado con las concentraciones al 5\%, lo que representa en proporción aumento de 30.85 y $41.10 \%$, respectivamente; sin embargo, al utilizar el ácido cítrico al 10\% como catalizador el tamaño de las microesferas se redujo en $75.32 \%$ con respecto a la concentración del 5\%; esa misma tendencia fue reportada por Velázquez-Maldonado et al. (2019).

Las microesferas en la superficie del biocarbón de ápices de caña de azúcar están asociadas con la descomposición de la celulosa. Román et al. (2012) indican que la recombinación de productos de carbono a partir de reacciones de descarboxilación, así como la ruptura de la celulosa, pueden formar nuevas estructuras de microesferas. En este sentido, el tamaño de las microesferas de carbón y la porosidad, dependen de la materia prima, catalizadores y técnica empleada en la elaboración del biocarbón. Por las propiedades físicas de su estructura y las características químicas del biocarbón de ápices de caña de azúcar, su incorporación al suelo como enmienda podría mejorar sus propiedades físicas, químicas y biológicas debido al incremento en la aireación, adsorción de nutrimentos e incremento poblacional de hongos micorrícicos (Velázquez-Maldonado et al., 2019; Singh et al., 2018; Escalante-Rebolledo et al., 2016).

Con los resultados obtenidos en biocarbón de ápices de caña de azúcar, se sugiere realizar investigaciones para determinar las características químicas, así como para conocer la estructura de biocarbones cuando se pretende usar nuevos materiales vegetales para su uso potencial como mejorador de suelos agrícolas, debido a que sus propiedades dependen principalmente de la materia prima empleada para elaborar el biocarbón.

\section{CONCLUSIONES}

Los mayores rendimientos del biocarbón de ápices de caña de azúcar elaborado mediante carbonización hidrotérmica (34.9-37.8\%) se obtuvieron con la adición de ácido cítrico a $10 \%$, y ácido maleico a 5 y $10 \%$ como catalizadores. En general, por la concentración nutrimental total y extraíble del biocarbón, su conductividad eléctrica (0.30-0.46 dS m $\left.{ }^{-1}\right)$, así como su estructura con microesferas, el biocarbón de ápices de caña de azúcar podría servir como mejorador de suelos, especialmente en suelos alcalinos debido al $\mathrm{pH}$ ácido del biocarbón producido.

\section{DECLARACIÓN DE ÉTICA}

No aplicable.

\section{CONSENTIMIENTO PARA PUBLICACIÓN}

No aplicable.

\section{DISPONIBILIDAD DE DATOS}

Los conjuntos de datos utilizados o analizados durante el estudio actual están disponibles del autor correspondiente a solicitud razonable.

\section{CONFLICTO DE INTERESES}

Los autores declaran que no tienen intereses en competencia.

\section{FONDOS}

No aplicable.

\section{CONTRIBUCIÓN DE LOS AUTORES}

Conceptualización: P.J.L., J.A.H. y C.A.P.C. Metodología, P.J.L., C.A.P.C. y J.A.H. Análisis formal: I.A.T. y E.S.P. Investigación: C.A.P.C., D.G.S., 
V.L.M., R.B.M. y R.C.B. Recursos: P.J.L. y J.A.H. Escritura: preparación del borrador original: C.A.P.C. y P.J.L. Escritura: revisión y edición: C.A.P.C, P.J.L., J.A.H., I.A.T., E.S.P., D.G.S., V.L.M., R.B.M. y R.C.B. Supervisión: P.J.L y J.A.H.

\section{AGRADECIMIENTOS}

El primer autor agradece al Consejo de Ciencia y Tecnología (CONACYT) por la beca otorgada para sus estudios de Doctorado en Ciencias Agropecuarias y Desarrollo Rural (becario: 243072; CVU: 260303).

\section{LITERATURA CITADA}

Adeyemi, T. O. A., \& Idowu, O. D. (2017). Biochar: promoting crop yield, improving soil fertility, mitigating climate change and restoring polluted soils. World News of Natural Sciences, 8, 27-36.

Alcántar-González, G., \& Trejo-Téllez, L. I. (2012). Nutrición de cultivos. Madrid, España: Paraninfo. ISBN 13: 9789687462486

Bento, L. R., Castro, A. J. R., Moreira, A. B., Ferreira, O. P., Bisinoti, M. C., \& Melo, C. A. (2019). Release of nutrients and organic carbon in different soil types from hydrochar obtained using sugarcane bagasse and vinasse. Geoderma, 334, 24-32. https://doi.org/10.1016/j.geoderma.2018.07.034

Blanco-Canqui, H. (2017). Biochar and soil physical properties. Soil Science Society of America Journal, 81, 687-711. https:// doi.org/10.2136/sssaj2017.01.0017

Concilco A., E., Moreno-Reséndez, A., García-Carrillo, M., Quiroga-Garza, H. M., \& García, O. Á. (2018). Influencia del biocarbón aplicado al suelo sobre atributos de rendimiento y calidad de avena forrajera. Terra Latinoamericana, 36(3), 221228. https://doi.org/10.28940/terra.v36i3.375

Cottenie, A. (1994). Workshop on Standardization of Analytical Methods for Manure, Soil Plant and Water. Commission European Communities. FAO Soils Bulletin 38/2. 28-33.

Chaparro-Garnica, J., Mostazo-López, M. J., Salinas-Torres, D., Morallon, E., \& Cazorla-Amorós, D. (2020). Residuos de biomasa como plataforma para obtener materiales carbonosos porosos mediante carbonización hidrotermal en presencia de $\mathrm{H}_{3} \mathrm{PO}_{4}$. Boletín del Grupo Español del Carbón, 55, 22-27.

Cho, M. S., Meng, L., Song, J. H., Han, S. H., Bae, K., \& Park, B. B. (2017). The effects of biochars on the growth of Zelkova serrata seedlings in a containerized seedling production system. Forest Science and Technology, 13(1), 25-30. https:// doi.org/10.1080/21580103.2017.1287778

Escalante-Rebolledo, A., Pérez-López, G., Hidalgo-Moreno, C., López-Collado, J., Campos-Alves, J., Valtierra-Pacheco, E., \& Etchevers-Barra, J. (2016). Biocarbón (biochar) I: Naturaleza, historia, fabricación y uso en el suelo. Terra Latinoamericana, 34(3), 367-382.

Gallo-Saravia, M., Lugo-Sierra, L., \& Barrera-Zapata, R. (2018). Evaluación de biochar como alternativa de sustrato en cultivos de tomate. Scientia et Technica, 23(2), 300-306. https://doi. org/10.22517/23447214.17691
Guerrero-Peña, A., Gómez-Merino, F. C., Hernández-Cázares, A. S., Salinas-Ruiz, J., \& Velasco-Velasco, J. (2017). Residuos orgánicos de la agroindustria azucarera: retos y oportunidades. Agro Productividad, 10(11), 99-104.

Ibarrola, R., Evar, B., \& Reay, D. (2013). Comercialización de biocarbón (biochar) en México. Definición del contexto para un programa de investigación multidisciplinario. Consultado el 01 de noviembre, 2020, desde http://rembio.org.mx/wpcontent/uploads/2014/10/Comercializacion_de_Biochar_en_ Mexico.pdf

IBI (International Biochar Initiative). (2015). Standardized product definition and product testing guidelines for biochar that is used in soil. Consultado el 01 de noviembre, 2020, desde http://www.biochar-international.org/characterizationstandard

Lynam, J. G., Toufic R., M., Vasquez, V. R., \& Coronella, C. J. (2012). Effect of salt addition on hydrothermal carbonization of lignocellulosic biomass. Fuel, 99, 271-273. https://doi. org/10.1016/j.fuel.2012.04.035

Medina O., L. E., \& Medina O., I. N. (2018). Prototipo autotérmico móvil para la producción de biocarbón con biomasa de esquilmos de aguacate. Terra Latinoamericana, 36(2), 121129. https://doi.org/10.28940/terra.v36i2.217

Orozco-Gutiérrez, G., \& Lira-Fuentes, R. C. (2020). Elaboración de biocarbón para el aprovechamiento de residuos proveniente de las podas de bambú (Guadua angustifolia). Revista Mexicana de Agroecosistemas, 7(1), 1-9.

Ortiz-Laurel, H., Salgado-García, S., Castelán-Estrada, M., \& Córdova-Sánchez, S. (2012). Perspectivas de la cosecha de la caña de azúcar cruda en México. Revista Mexicana de Ciencias Agrícolas, 3(Esp. 4), 767-773.

Quesada-Kimsey, J. (2012). La carbonización de residuos biomásicos: una exploración con perspectivas emocionantes. Revista Tecnología en Marcha, 25(5), 14-21. https://doi. org/10.18845/tm.v25i5.465

Román, S., Nabais, J. M. V., Laginhas, C., Ledesma, B., \& González, J. F. (2012). Hydrothermal carbonization as an effective way of densifying the energy content of biomass. Fuel Processing Technology, 103, 78-83. https://doi.org/10.1016/j. fuproc.2011.11.009

SAS Institute. (2004). SAS/STAT User's Guide. Release 9.1.3. SAS Institute. Cary, NC, USA.

Schlegel, M. M., Ibrahim, B., Kipping-Rössel, D., Ortiz-Laurel, H., \& Fras, J. (2018). Generación de biocarbón a partir del material sólido en la hidrólisis aeróbico-microbiológica. Agro Productividad, 11(11), 27-33. http://dx.doi.org/10.32854/ agrop.v11i11.1279

Sevilla, M., \& Titirici, M. M. (2012). Hydrothermal carbonization: a greener route towards the synthesis of advanced carbon materials. Boletín del Grupo Español Carbón, 25, 7-17.

SIAP (Servicio de Información Agroalimentaria y Pesquera). (2020). Anuario Estadístico de la Producción Agrícola. Consultado el 02 de noviembre, 2020, desde https://nube.siap. gob.mx/cierreagricola/

Silva, C. C., Melo, C. A., Soares-Junior, F. H., Moreira, A. B., Ferreira, O. P., \& Bisinoti, M. C. (2017). Effect of the reaction medium on the immobilization of nutrients in hydrochars obtained using sugarcane industry residues. Bioresource Technology, 237, 213-221. http://dx.doi.org/10.1016/j. biortech.2017.04.004 
Singh, A., Singh, A. P., \& Purakayastha, T. J. (2018). Characterization of biochar and their influence on microbial activities and potassium availability in an acid soil. Archives of Agronomy and Soil Science, 65(9), 1302-1315. https://doi.org/ 10.1080/03650340.2018.1563291

Stadler-Kaulich, N., \& Perteguer, A. H. (2018). Más Allá de la agroforestería. El biocarbón activado y la madera rameal fragmentada: utilización y preparación en Mollesnejta. Revista Acta Nova, 8(4), 572-592

Steiner, C. (2016). Considerations in biochar characterization. In: M. Guo, Z. He, \& S. M. Uchimiya (Eds.). Agricultural and Environmental Applications of Biochar: Advances and Barriers (pp. 87-99). Hoboken, NJ, USA: SSSA Special Publication, John Wiley \& Sons. https://doi.org/10.2136/ sssaspecpub63.2014.0038.5

Varela-Milla, O., Rivera, E. B., Huang, W. J., Chien, C. C., \& Wang, Y. M. (2013). Agronomic properties and characterization of rice husk and wood biochars and their effect on the growth of water spinach in a field test. Journal of Soil Science and Plant Nutrition, 13(2), 251-266. http://dx.doi.org/10.4067/S071895162013005000022

Velázquez-Machuca, M. A., Equihua-Soriano, J. R., VenegasGonzález, J., Montañez-Soto, J. L., Pimentel-Equihua, J. L., \& Muñoz-Navia, M. (2019). Caracterización física y química de biochar de lodos residuales. Terra Latinoamericana, 37(3), 243-251. https://doi.org/10.28940/terra.v37i3.409
Velázquez-Maldonado, J., Juárez-López, P., Anzaldo-Hernández, J., Alejo-Santiago, G., Valdez-Aguilar, L. A., Alia-Tejacal, I., López-Martínez, V., Pérez-Arias, G. A., \& Guillén-Sánchez, D. (2019). Concentración nutrimental de biocarbón de cascarilla de arroz. Revista Fitotecnia Mexicana, 42(2), 129-136.

Wang, J., \& Wang, S. (2019). Preparation, modification and environmental application of biochar: A review. Journal of Cleaner Production, 227(1), 1002-1022. https://doi. org/10.1016/j.jclepro.2019.04.282

Xu, Y., Xia, M., Jiang, Y., Li, F., \& Xue, B. (2018). Opal promotes hydrothermal carbonization of hydroxypropyl methyl cellulose and formation of carbon nanospheres. RSC Advances, 8, 20095-20107. https://doi.org/10.1039/c8ra01138a

Zhang, Y. F., Dai, J. M., Guo, H., Shi, S., Yan, Z. F., \& Hou, W. S. (2020). A comparative study of carbon microsphere preparation by the hydrothermal carbonization of waste cotton fibers, viscose fibers and Avicel. New Carbon Materials, 35(3): 286294. https://doi.org/10.1016/S1872-5805(20)60490-5

Zheng, W., Holm, N., \& Spokas, K. A. (2016). Research and application of biochar in North America. In M. Guo, Z. He, \& S. M. Uchimiya, (Eds.). Agricultural and Environmental Applications of Biochar: Advances and Barriers (pp. 475-494). Hoboken, NJ, USA: SSSA Special Publication, John Wiley \& Sons. https://doi.org/10.2136/sssaspecpub63.2014.0053 